\title{
Two-Dimensional Microwave Fourier Transform Spectroscopy
}

\author{
W. Stahl, E. Fliege, and H. Dreizler \\ Abteilung Chemische Physik im Institut für Physikalische Chemie der Universität Kiel
}

Z. Naturforsch. 39 a, 858-864 (1984); received June 27, 1984

Herrn Prof. Dr. H. D. Rudolph, Ulm, zum 60. Geburtstag gewidmet

We performed a pulse sequence experiment utilizing a technique, which is comparable with two-dimensional NMR-spectroscopy. Some theoretical considerations based on the three-level Bloch equations are given. This double resonance technique may be useful for the assignment of complicated spectra.

\section{Introduction}

In 1982 we have shown for the first time that it is possible to perform a double resonance experiment with a microwave Fourier transform (MWFT) spectrometer [1]. A continuous pump microwave was used and a very accurate determination of the pump transition frequency was possible. The method was not useful for detecting pump transitions when the pump frequency was a few $\mathrm{MHz}$ off-resonant, which does not agree with the experience in MW double resonance spectroscopy in the frequency domain. Somewhat later Andrews et al. [2] performed a pulse sequence double resonance experiment. They worked with a continuous probe microwave and applied two pump pulses with a variable delay in between. The time resolved and Fourier transformed response of the system was plotted as a function of the time delay between the pump pulses. For the special case of a resonant pump frequency this experiment is similar to one which has been carried out by Bomsdorf and Dreizler [3].

In this paper we describe a pure pulse sequence experiment where no continuous microwave is used. Instead we applied a signal pulse to the molecular system to provide for an initial polarisation of the signal transition. The maximum off-resonance of the pump frequency is comparable to the values reported in [2].

\section{Theory}

The basis of the theoretical considerations for this experiment is given by the system of three-level

Reprint requests to Prof. Dr. H. Dreizler, Institut für Physikalische Chemie der Universität Kiel, Olshausenstr. 40, D-2300 Kiel.
Bloch equations. In this paper we presuppose the knowledge of the nomenclature and the definitions of the used quantities as they are given in [3].

The pulse sequence and the three-level system which has been used in our experiment is shown in Figure $1^{*}$. At the beginning of the experiment a resonant signal pulse was generated. After a negligible delay of the order of $10 \mathrm{~ns}$ the first pump pulse followed and after a delay of the length $\tau$ a second pump pulse equal in frequency, intensity and length to the first one was produced. The measurement of a transient signal in the frequency range of the signal transition began about $10 \mathrm{~ns}$ after the end of the second pump pulse. The frequency of the pump microwave was chosen to be near resonant. During the experiment the delay between both pulses was incremented stepwise, all other delays and pulse lengths remained unchanged.

For a theoretical treatment of the behavior of the system we will consider the different phases of the experiment individually. We refer to Eq. (3) of [3].

\section{Phase 1: Resonant signal pulse}

At the beginning $t_{0}=0$ of the signal pulse the system is in thermal equilibrium with $P_{i j}=0$, $Q_{i j}=0$ and $\Delta N_{i j}=\Delta N_{i j}^{0} ; i, j=a, b, c$.

Then a resonant signal microwave of the frequency $\omega_{a b}$ with $\Delta \omega_{a b}=0$ is applied to the system. This case can be treated with the two-level Bloch equations derived from Eq. (3) of [3] with $\varepsilon_{a b} \neq 0$, $\varepsilon_{b c}=0$. We assume that relaxation can be neglected, as $t_{1}-t_{0} \ll T_{2}, \gamma_{z}, \gamma_{y}$ and take a $\pi / 2$-pulse to simplify the solutions. At the end $t_{1}$ of the pulse they

* Observe that in [3] pump and signal are exchanged.

0340-4811/84/0900-0858 \$01.30/0. - Please order a reprint rather than making your own copy. 

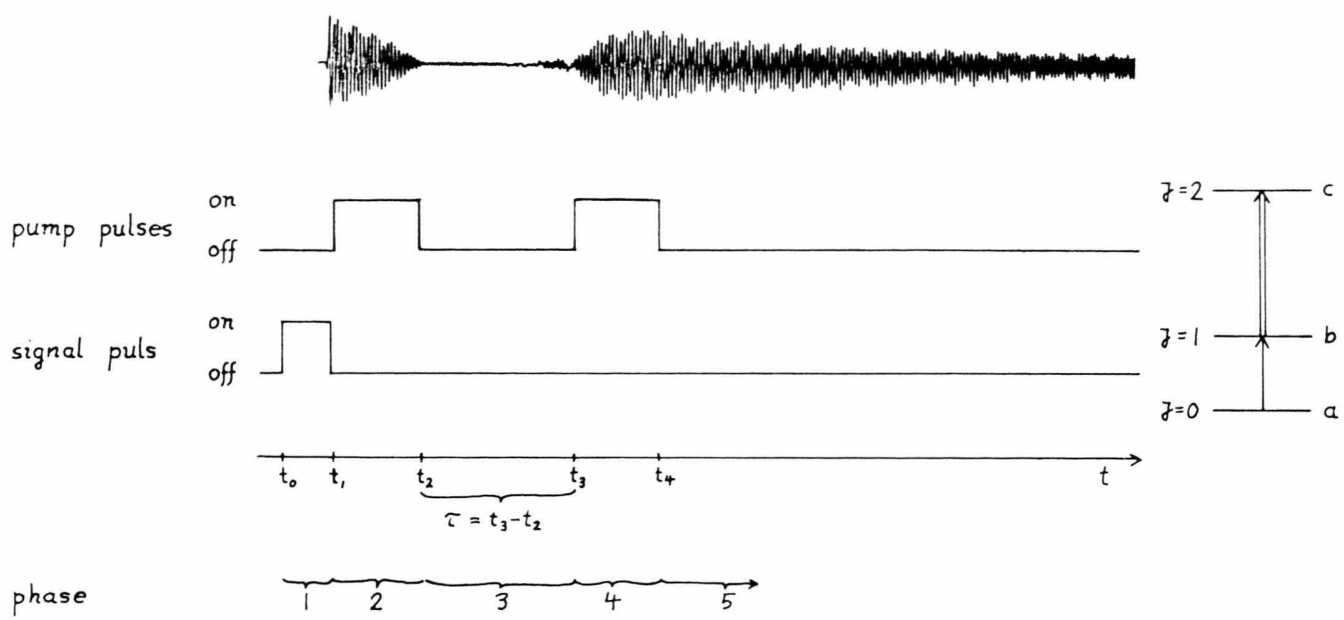

Fig. 1. Pulse sequence of our experiment and level scheme of carbonylsulfide, OCS. Observation of the transient decay in phase 5.

are given by:

$$
\begin{aligned}
& P_{a b}\left(t_{1}\right)=0, \\
& Q_{a b}\left(t_{1}\right)=\left(-\frac{\hbar \varkappa_{a b}}{4}\right) \Delta N_{a b}^{0}, \\
& \Delta N_{a b}\left(t_{1}\right)=0
\end{aligned}
$$

with

$$
\varkappa_{a b}=\frac{2 \mu_{a b}}{\hbar} .
$$

The preparation of the system by a $\pi / 2$-signal pulse causes a nonzero-value for $Q_{a b}$ of the $a b$-polarisation. All other variables are zero.

\section{Phase 2: Off-resonant pump pulse}

Phase 2 can be described by a system of differential equations which is obtained from the general three-level Bloch equations (3) of [3] with $\varepsilon_{a b}=0$ and $\Delta \omega_{a b}=0$

$$
\begin{aligned}
\gamma_{x} \Delta N_{b c}+\gamma_{w} \Delta N_{a b}-4 \frac{\varepsilon_{b c}}{\hbar} Q_{b c} \\
-\left(\Delta N_{a b}^{0} \gamma_{w}+\Delta N_{b c}^{0} \gamma_{x}\right)=-\Delta \dot{N}_{b c},
\end{aligned}
$$

$$
\gamma=\Delta N_{b c}+\gamma_{y} \Delta N_{a b}+2 \frac{\varepsilon_{b c}}{\hbar} Q_{b c}
$$$$
-\left(\Delta N_{a b}^{0} \gamma_{y}+\Delta N_{b c}^{0} \gamma_{z}\right)=-\Delta \dot{N}_{a b},
$$

$\frac{1}{T_{2}^{b c}} P_{b c}+\Delta \omega_{b c} Q_{b c} \quad=-\dot{P}_{b c}$,

$$
\begin{aligned}
& \mu_{b c}^{2} \frac{\varepsilon_{b c}}{\hbar} \Delta N_{b c}-\Delta \omega_{b c} P_{b c}+\frac{1}{T_{2}^{b c}} Q_{b c}=-\dot{Q}_{b c}, \\
& \frac{1}{T_{2}^{a c}} P_{a c}+\Delta \omega_{a c} Q_{a c}-\mu_{b c}^{2} \frac{\varepsilon_{b c}}{\hbar} Q_{a b}=-\dot{P}_{a c}, \\
& -\Delta \omega_{a c} P_{a c}+\frac{1}{T_{2}^{a c}} Q_{a c}+\mu_{b c}^{2} \frac{\varepsilon_{b c}}{\hbar} P_{a b}=-\dot{Q}_{a c}, \\
& -\frac{\varepsilon_{b c}}{\hbar} Q_{a c}+\frac{1}{T_{2}^{a b}} P_{a b}+\Delta \omega_{a b} Q_{a b}=-\dot{P}_{a b}, \\
& \frac{\varepsilon_{b c}}{\hbar} P_{a c}-\Delta \omega_{a b} P_{a b}+\frac{1}{T_{2}^{a b}} Q_{a b}=-\dot{Q}_{a b} .
\end{aligned}
$$

The system of the three-level Bloch equations (Eq. (3) of [3]) is separated into two subsystems of coupled differential equations. The system $(3 \mathrm{a}-\mathrm{d})$ contains information on the $b c$-polarisation. We shall not report on its solutions here because they are not needed for the further considerations.

Neglecting relaxational terms with the assumption $t_{2}-t_{1} \ll T_{2}, \quad \gamma_{i}, i=x, y, z, w$ we could solve $(4 \mathrm{a}-\mathrm{d})$ by Laplace-transformation technique [4].

$$
\begin{aligned}
P_{a c}\left(t_{2}\right)= & Q_{a b}\left(t_{1}\right) \frac{\mu_{b c} \varkappa_{b c} \varepsilon_{b c}}{2} \\
& \cdot\left[\frac{\Omega_{1}^{2}-\chi_{b c}^{2} \varepsilon_{b c}^{2} / 4}{\left(\Omega_{1}^{2}-\Omega_{2}^{2}\right) \Omega_{1}} \sin \Omega_{1}\left(t_{2}-t_{1}\right)\right. \\
& \left.+\frac{\Omega_{2}^{2}-\varkappa_{b c}^{2} \varepsilon_{b c}^{2} / 4}{\left(\Omega_{2}^{2}-\Omega_{1}^{2}\right) \Omega_{2}} \sin \Omega_{2}\left(t_{2}-t_{1}\right)\right],
\end{aligned}
$$




$$
\begin{aligned}
Q_{a c}\left(t_{2}\right)= & -Q_{a b}\left(t_{1}\right) \frac{\mu_{b c} \varkappa_{b c} \varepsilon_{b c} \Delta \omega_{b c}}{2\left(\Omega_{1}^{2}-\Omega_{2}^{2}\right)} \\
& \cdot\left[\cos \Omega_{1}\left(t_{2}-t_{1}\right)-\cos \Omega_{2}\left(t_{2}-t_{1}\right)\right], \quad(5 \mathrm{~b}) \\
P_{a b}\left(t_{2}\right)= & -Q_{a b}\left(t_{1}\right) \frac{x_{b c}^{2} \varepsilon_{b c}^{2} \Delta \omega_{b c}}{4\left(\Omega_{1}^{2}-\Omega_{2}^{2}\right)} \\
& \cdot\left[\frac{1}{\Omega_{1}} \sin \Omega_{1}\left(t_{2}-t_{1}\right)-\frac{1}{\Omega_{2}} \sin \Omega_{2}\left(t_{2}-t_{1}\right)\right], \\
Q_{a b}\left(t_{2}\right)= & Q_{a b}\left(t_{1}\right)\left[\frac{\Omega_{1}^{2}-\chi_{b c}^{2} \varepsilon_{b c}^{2} / 4-\Delta \omega_{b c}^{2}}{\Omega_{1}^{2}-\Omega_{2}^{2}} \cos \Omega_{1}\left(t_{2}-t_{1}\right)\right. \\
& \left.+\frac{\Omega_{2}^{2}-\chi_{b c}^{2} \varepsilon_{b c}^{2} / 4-\Delta \omega_{b c}^{2}}{\Omega_{2}^{2}-\Omega_{1}^{2}} \cos \Omega_{2}\left(t_{2}-t_{1}\right)\right](5 \mathrm{~d})
\end{aligned}
$$

with

For (5) the analogous consideration is more complicated. For a certain time $t_{2}-t_{1}$ with

$$
t_{\alpha}-t_{1}>t_{2}-t_{1}>t_{\beta}-t_{1}
$$

where

$$
\Omega_{1}\left(t_{x}-t_{1}\right)=\pi / 2
$$

and

$$
\Omega_{2}\left(t_{\beta}-t_{1}\right)=\pi / 2
$$

and always

$$
\Omega_{1}<\Omega_{2}
$$

$Q_{a b}\left(t_{2}\right)$ can be adjusted to zero, which is observable in the experiment (see Figure 1). $P_{a c}\left(t_{2}\right)$ is then near to its maximum.

$$
\begin{aligned}
& \Omega_{1}=\sqrt{\frac{x_{b c}^{2} \varepsilon_{b c}^{2}}{4}+\frac{\Delta \omega_{b c}^{2}}{2}-\sqrt{\left(\frac{x_{b c}^{2} \varepsilon_{b c}^{2}}{4}+\frac{\Delta \omega_{b c}^{2}}{2}\right)^{2}-\left(\frac{x_{b c}^{2} \varepsilon_{b c}^{2}}{4}\right)^{2}}}, \\
& \Omega_{2}=\sqrt{\frac{x_{b c}^{2} \varepsilon_{b c}^{2}}{4}+\frac{\Delta \omega_{b c}^{2}}{2}+\sqrt{\left(\frac{x_{b c}^{2} \varepsilon_{b c}^{2}}{4}+\frac{\Delta \omega_{b c}^{2}}{2}\right)^{2}-\left(\frac{x_{b c}^{2} \varepsilon_{b c}^{2}}{4}\right)^{2}}},
\end{aligned}
$$

$x_{b c}=2 \mu_{b c} / \hbar$

and

$$
\Delta \omega_{b c}=\Delta \omega_{a c} \quad \text { as } \quad \Delta \omega_{a b}=0 .
$$

The solutions (5) are valid for short pump pulses and the initial conditions given by (1). For resonant pump, $\Delta \omega_{b c}=0$ or for strong off resonant pump $x_{b c}^{2} \varepsilon_{b c}^{2} / 2 \gg \Delta \omega_{b c}^{2}$

$$
\Omega_{1}=\Omega_{2}=\chi_{b c} \varepsilon_{b c} / 2=\frac{\mu_{b c} \varepsilon_{b c}}{\hbar} .
$$

For this case the solutions (5) converge to

$$
\begin{aligned}
P_{a c}\left(t_{2}\right) & =Q_{a b}\left(t_{1}\right) \mu_{b c} \sin \frac{\mu_{b c} \varepsilon_{b c}}{\hbar}\left(t_{2}-t_{1}\right), \\
Q_{a c}\left(t_{2}\right) & =0, \\
P_{a b}\left(t_{2}\right) & =0, \\
Q_{a b}\left(t_{2}\right) & =Q_{a b}\left(t_{1}\right) \cos \frac{\mu_{b c} \varepsilon_{b c}}{\hbar}\left(t_{2}-t_{1}\right) .
\end{aligned}
$$

Observing that in this experiment pump and signal are exchanged with respect to [3] (7a) corresponds to (11) of [3] and (7d) to (11 a) of [3].

From (7) it is immediately obvious that for $\frac{\mu_{b c} \varepsilon_{b c}}{\hbar_{1}}\left(t_{2}-t_{1}\right)=\frac{\pi}{2}$, a $\frac{\pi}{2}$-pump pulse, $P_{a c}\left(t_{\pi / 2}\right)$ is at a maximum.
Phase 3: Time interval between pump pulses

During the time interval of the length $\tau=t_{3}-t_{2}$ between both pump pulses no external microwave field is present; $\varepsilon_{a b}=\varepsilon_{b c}=0$. The initial conditions are generally

$$
\begin{array}{lll}
P_{a c}\left(t_{2}\right) \neq 0, & Q_{a c}\left(t_{2}\right) \neq 0, & P_{a b}\left(t_{2}\right) \neq 0, \\
Q_{a b}\left(t_{2}\right) \neq 0, & P_{b c}\left(t_{2}\right) \neq 0, & Q_{b c}\left(t_{2}\right) \neq 0,
\end{array}
$$

but $Q_{a b}\left(t_{2}\right)$ can be adjusted to zero.

The system of three-level Bloch equations is given by

$$
\begin{aligned}
& \gamma_{x} \Delta N_{b c}+\gamma_{w} \Delta N_{a b} \\
&-\left(\Delta N_{a b}^{0} \gamma_{w}+\Delta N_{b c}^{0} \gamma_{x}\right)=-\Delta \dot{N}_{b c}, \\
& \gamma_{2} \Delta N_{b c}+\gamma_{y} \Delta N_{a b} \\
&-\left(\Delta N_{a b}^{0} \gamma_{x}+\Delta N_{b c}^{0} \gamma_{z}\right)=-\Delta \dot{N}_{a b}, \\
& \frac{1}{T_{2}^{b c}} P_{b c}+\Delta \omega_{b c} Q_{b c}=-\dot{P}_{b c}, \\
&-\Delta \omega_{b c} P_{b c}+\frac{1}{T_{2}^{b c}} Q_{b c}=-\dot{Q}_{b c}, \\
& \frac{1}{T_{2}^{a c}} P_{a c}+\Delta \omega_{a c} Q_{a c}=-\dot{P}_{a c}, \\
&-\Delta \omega_{a c} P_{a c}+\frac{1}{T_{2}^{a c}} Q_{a c}=-\dot{Q}_{a c},
\end{aligned}
$$


W. Stahl et al. · Two-Dimensional Microwave Fourier Transform Spectroscopy

$$
\begin{aligned}
& \frac{1}{T_{2}^{a b}} P_{a b}+\Delta \omega_{a b} Q_{a b}=-\dot{P}_{a b}, \\
& -\Delta \omega_{a b} P_{a b}+\frac{1}{T_{2}^{a b}} Q_{a b}=-\dot{Q}_{a b} .
\end{aligned}
$$

The whole system (8) consists of four subsystems containing two coupled differential equations each.

The solutions can easily be obtained. Under consideration of the general initial conditions and assuming resonant signal pulses $\left(\Delta \omega_{a b}=0\right)$ they are given for $(8 \mathrm{e}-\mathrm{h})$ by

$$
\begin{array}{rlr}
P_{a c}(\tau)= & P_{a c}\left(t_{3}\right)=e^{-\left(t_{3}-t_{2}\right) / T_{2}^{a c}}\left(P_{a c}\left(t_{2}\right) \cos \Delta \omega_{b c}\left(t_{3}-t_{2}\right)\right. \\
& \left.-Q_{a c}\left(t_{2}\right) \sin \Delta \omega_{b c}\left(t_{3}-t_{2}\right)\right), & (9 \mathrm{a}) \\
Q_{a c}(\tau)= & Q_{a c}\left(t_{3}\right)=e^{-\left(t_{3}-t_{2}\right) / T_{2}^{a c}}\left(Q_{a c}\left(t_{2}\right) \cos \Delta \omega_{b c}\left(t_{3}-t_{2}\right)\right. \\
& \left.+P_{a c}\left(t_{2}\right) \sin \Delta \omega_{b c}\left(t_{3}-t_{2}\right)\right), & (9 \mathrm{~b}) \\
P_{a b}(\tau)= & P_{a b}\left(t_{3}\right)=e^{-\left(t_{3}-t_{2}\right) / T_{2}^{a b}} P_{a b}\left(t_{2}\right), & (9 \mathrm{c}) \\
Q_{a b}(\tau)= & Q_{a b}\left(t_{3}\right)=e^{-\left(t_{3}-t_{2}\right) / T_{2}^{a b}} Q_{a b}\left(t_{2}\right) . & (9 \mathrm{~d})
\end{array}
$$

Has $Q_{a b}\left(t_{2}\right)$ been adjusted to zero $(9 \mathrm{~d})$ specialises to $Q_{a b}(\tau)=0$.
As in the case of phase 2 we are not interested in the solution for the $b c$-polarisation.

The time dependent behavior of the $a c$-polarisation can be described as a precession of the Bloch vector $\left(P_{a c}, Q_{a c}\right)$ with the off-resonance pump frequency $\Delta \omega_{b c}=\Delta \omega_{a c}$ under simultaneous relaxation with the relaxation time $T_{2}^{a c}$. Neglecting relaxation $P_{a c}(\tau)$ and $Q_{a c}(\tau)$ are periodic functions with the period $\Delta \omega_{b c} . P_{a b}(\tau)$ and $Q_{a b}(\tau)$ are not periodic.

Phase 4: Second off-resonant pump pulse

Phase 4 can be described by the same system of Bloch equations (3), (4) as in the case of phase 2. This time we are interested in the solution for the $a b$-polarisation only because all other quantities cannot be detected by a measurement in our experiment. The initial conditions for $\tau=t_{3}-t_{2}$ are

$P_{a c}(\tau) \neq 0, Q_{a c}(\tau) \neq 0, P_{a b}(\tau) \neq 0, Q_{a b}(\tau) \neq 0$.

with $\pi / 2$-pump pulse $Q_{a b}(\tau)$ can be made zero.

The solutions of (4) with neglected relaxation during the pulses are

$$
\begin{aligned}
& P_{a c}\left(t_{4}, \tau\right)=P_{a c}(\tau)\left[\frac{\Omega_{1}^{2}-\chi_{b c}^{2} \varepsilon_{b c}^{2} / 4}{\Omega_{1}^{2}-\Omega_{2}^{2}} \cos \Omega_{1}\left(t_{4}-t_{3}\right)+\frac{\Omega_{2}^{2}-x_{b c}^{2} \varepsilon_{b c}^{2} / 4}{\Omega_{2}^{2}-\Omega_{1}^{2}} \cos \Omega_{2}\left(t_{4}-t_{3}\right)\right] \\
& -Q_{a c}(\tau) \frac{\Delta \omega_{b c}}{\Omega_{1}^{2}-\Omega_{2}^{2}}\left(\Omega_{1} \sin \Omega_{1}\left(t_{4}-t_{3}\right)-\Omega_{2} \sin \Omega_{2}\left(t_{4}-t_{3}\right)\right) \\
& -P_{a b}(\tau) \frac{\mu_{b c} \varkappa_{b c} \varepsilon_{b c} \Delta \omega_{b c}}{2\left(\Omega_{1}^{2}-\Omega_{2}^{2}\right)}\left(\cos \Omega_{1}\left(t_{4}-t_{3}\right)-\cos \Omega_{2}\left(t_{4}-t_{3}\right)\right) \\
& +Q_{a b}(\tau) \frac{\mu_{b c} \varkappa_{b c} \varepsilon_{b c}}{2\left(\Omega_{1}^{2}-\Omega_{2}^{2}\right)}\left[\left(\Omega_{1}-\varkappa_{b c}^{2} \varepsilon_{b c}^{2} / 4 \Omega_{1}\right) \sin \Omega_{1}\left(t_{4}-t_{3}\right)\right. \\
& \left.-\left(\Omega_{2}-x_{b c}^{2} \varepsilon_{b c}^{2} / 4 \Omega_{2}\right) \sin \Omega_{2}\left(t_{4}-t_{3}\right)\right] ; \\
& Q_{a c}\left(t_{4}, \tau\right)=P_{a c}(\tau) \frac{\Delta \omega_{b c}}{\Omega_{1}^{2}-\Omega_{2}^{2}}\left(\Omega_{1} \sin \Omega_{1}\left(t_{4}-t_{3}\right)-\Omega_{2} \sin \Omega_{2}\left(t_{4}-t_{3}\right)\right) \\
& +Q_{a c}(\tau)\left[\frac{\Omega_{1}^{2}-x_{b c}^{2} \varepsilon_{b c}^{2} / 4}{\Omega_{1}^{2}-\Omega_{2}^{2}} \cos \Omega_{1}\left(t_{4}-t_{3}\right)+\frac{\Omega_{2}^{2}-x_{b c}^{2} \varepsilon_{b c}^{2} / 4}{\Omega_{2}^{2}-\Omega_{1}^{2}} \cos \Omega_{2}\left(t_{4}-t_{3}\right)\right] \\
& -P_{a b}(\tau) \frac{\mu_{b c} \varkappa_{b c} \varepsilon_{b c}}{2\left(\Omega_{1}^{2}-\Omega_{2}^{2}\right)}\left[\left(\Omega_{1}-\varkappa_{b c}^{2} \varepsilon_{b c}^{2} / 4 \Omega_{1}\right) \sin \Omega_{1}\left(t_{4}-t_{3}\right)\right. \\
& \left.-\left(\Omega_{2}-\chi_{b c}^{2} \varepsilon_{b c}^{2} / 4 \Omega_{2}\right) \sin \Omega_{2}\left(t_{4}-t_{3}\right)\right] \\
& -Q_{a b}(\tau) \frac{\mu_{b c} \varkappa_{b c} \varepsilon_{b c} \Delta \omega_{b c}}{2\left(\Omega_{1}^{2}-\Omega_{2}^{2}\right)}\left(\cos \Omega_{1}\left(t_{4}-t_{3}\right)-\cos \Omega_{2}\left(t_{4}-t_{3}\right)\right) ;
\end{aligned}
$$




$$
\begin{aligned}
P_{a b}\left(t_{4}, \tau\right)= & -P_{a c}(\tau) \frac{\varepsilon_{b c} \Delta \omega_{b c}}{\left(\Omega_{1}^{2}-\Omega_{2}^{2}\right) \hbar}\left(\cos \Omega_{1}\left(t_{4}-t_{3}\right)-\cos \Omega_{2}\left(t_{4}-t_{3}\right)\right) \\
& +Q_{a c}(\tau) \frac{\varepsilon_{b c}}{\hbar_{1}}\left[\frac{\Omega_{1}-x_{b c}^{2} \varepsilon_{b c}^{2} / 4 \Omega_{1}}{\Omega_{1}^{2}-\Omega_{2}^{2}} \sin \Omega_{1}\left(t_{4}-t_{3}\right)+\frac{\Omega_{2}-x_{b c}^{2} \varepsilon_{b c}^{2} / 4 \Omega_{2}}{\Omega_{2}^{2}-\Omega_{1}^{2}} \sin \Omega_{2}\left(t_{4}-t_{3}\right)\right] \\
& +P_{a b}(\tau)\left[\frac{\Omega_{1}^{2}-x_{b c}^{2} \varepsilon_{b c}^{2} / 4-\Delta \omega_{b c}^{2}}{\Omega_{1}^{2}-\Omega_{2}^{2}} \cos \Omega_{1}\left(t_{4}-t_{3}\right)+\frac{\Omega_{2}^{2}-x_{b c}^{2} \varepsilon_{b c}^{2} / 4-\Delta \omega_{b c}^{2}}{\Omega_{2}^{2}-\Omega_{1}^{2}} \cos \Omega_{2}\left(t_{4}-t_{3}\right)\right] \\
& -Q_{a b}(\tau) \frac{x_{b c}^{2} \varepsilon_{b c}^{2} \Delta \omega_{b c}}{4\left(\Omega_{1}^{2}-\Omega_{2}^{2}\right)}\left[\frac{1}{\Omega_{1}} \sin \Omega_{1}\left(t_{4}-t_{3}\right)-\frac{1}{\Omega_{2}} \sin \Omega_{2}\left(t_{4}-t_{3}\right)\right] ; \\
Q_{a b}\left(t_{4}, \tau\right)= & -P_{a c}(\tau) \frac{\varepsilon_{b c}\left[\frac{\Omega_{1}-x_{b c}^{2} \varepsilon_{b c}^{2} / 4 \Omega_{1}}{\hbar_{l}} \sin \Omega_{1}^{2}\left(t_{4}-t_{3}^{2}\right)+\frac{\Omega_{2}-x_{b c}^{2} \varepsilon_{b c}^{2} / 4 \Omega_{2}}{\Omega_{2}^{2}-\Omega_{1}^{2}} \sin \Omega_{2}\left(t_{4}-t_{3}\right)\right]}{(10 \mathrm{c})]} \\
& -Q_{a c}(\tau) \frac{\varepsilon_{b c} \Delta \omega_{b c}}{\left(\Omega_{1}^{2}-\Omega_{2}^{2}\right) \hbar}\left(\cos \Omega_{1}\left(t_{4}-t_{3}\right)-\cos \Omega_{2}\left(t_{4}-t_{3}\right)\right) \\
& +P_{a b}(\tau) \frac{x_{b c}^{2} \varepsilon_{b c}^{2} \Delta \omega_{b c}}{4\left(\Omega_{1}^{2}-\Omega_{2}^{2}\right)}\left[\frac{1}{\Omega_{1}} \sin \Omega_{1}\left(t_{4}-t_{3}\right)-\frac{1}{\Omega_{2}} \sin \Omega_{2}\left(t_{4}-t_{3}\right)\right] \\
& +Q_{a b}(\tau)\left[\frac{\Omega_{1}^{2}-x_{b c}^{2} \varepsilon_{b c}^{2} / 4-\Delta \omega_{b c}^{2}}{\Omega_{1}^{2}-\Omega_{2}^{2}} \cos \Omega_{1}\left(t_{4}-t_{3}\right)+\frac{\Omega_{2}^{2}-x_{b c}^{2} \varepsilon_{b c}^{2} / 4-\Delta \omega_{b c}^{2}}{\Omega_{2}^{2}-\Omega_{1}^{2}} \cos \Omega_{2}\left(t_{4}-t_{3}\right)\right] .
\end{aligned}
$$

At a certain pulse length $t_{4}-t_{3}$ it is possible to regain $(a b)$-polarisation. The $(a b)$-polarisation determines the magnitude of the decay in phase 5 . This can be seen much easier for a resonant pump frequency, $\Delta \omega_{b c}=0$, where $(10 \mathrm{a}-\mathrm{d})$ can be simplified to

$$
\begin{aligned}
P_{a c}\left(t_{4}, \tau\right)= & P_{a c}(\tau) \cos \frac{\mu_{b c} \varepsilon_{b c}}{\hbar}\left(t_{4}-t_{3}\right) \\
& +Q_{a b}(\tau) \mu_{b c} \sin \frac{\mu_{b c} \varepsilon_{b c}}{\hbar}\left(t_{4}-t_{3}\right), \\
Q_{a c}\left(t_{4}, \tau\right)= & Q_{a c}(\tau) \cos \frac{\mu_{b c} \varepsilon_{b c}}{\hbar}\left(t_{4}-t_{3}\right) \\
& -P_{a b}(\tau) \mu_{b c} \sin \frac{\mu_{b c} \varepsilon_{b c}}{\hbar}\left(t_{4}-t_{3}\right), \\
P_{a b}\left(t_{4}, \tau\right)= & Q_{a c}(\tau) \frac{1}{\mu_{b c}} \sin \frac{\mu_{b c} \varepsilon_{b c}}{\hbar}\left(t_{4}-t_{3}\right) \\
& +P_{a b}(\tau) \cos \frac{\mu_{b c} \varepsilon_{b c}}{\hbar}\left(t_{4}-t_{3}\right), \\
Q_{a b}\left(t_{4}, \tau\right)= & -P_{a c}(\tau) \frac{1}{\mu_{b c}} \sin \frac{\mu_{b c} \varepsilon_{b c}}{\hbar}\left(t_{4}-t_{3}\right) \\
& +Q_{a b}(\tau) \cos \frac{\mu_{b c} \varepsilon_{b c}}{\hbar}\left(t_{4}-t_{3}\right) .
\end{aligned}
$$

As we pointed out in the treatment of phase 2 it is possible to adjust $Q_{a b}\left(t_{2}\right), P_{a b}\left(t_{2}\right)$ and $Q_{a c}\left(t_{2}\right)$ to zero in the case of a resonant pump. This assumption leads to

$$
\begin{array}{ll}
P_{a c}\left(t_{4}, \tau\right)=P_{a c}(\tau) \cos \frac{\mu_{b c} \varepsilon_{b c}}{\hbar}\left(t_{4}-t_{3}\right), & (12 \mathrm{a}) \\
Q_{a c}\left(t_{4}, \tau\right)=0, & (12 \mathrm{~b}) \\
P_{a b}\left(t_{4}, \tau\right)=0, & (12 \mathrm{c}) \\
Q_{a b}\left(t_{4}, \tau\right)=-P_{a c}(\tau) \frac{1}{\mu_{b c}} \sin \frac{\mu_{b c} \varepsilon_{b c}}{\hbar}\left(t_{4}-t_{3}\right) .
\end{array}
$$

When the pulse length of the second pump pulse is adjusted to

$$
\frac{\mu_{b c} \varepsilon_{b c}}{\hbar_{l}}\left(t_{4}-t_{3}\right)=\frac{\pi}{2} .
$$

$P_{a c}$ becomes zero and for $Q_{a b}$ a maximum value will be obtained. Its magnitude depends only on the length $\tau$ of the time interval between both pump pulses when constant pulse lengths are presupposed. Neglecting relaxation it is a periodic function (see (9)) with the period $\Delta \omega_{b c}$. In this way the off-resonance $\Delta \omega_{b c}$ of the pump and the relaxation time $T_{2}^{a c}$ become observables as the initial conditions 
$Q_{a b}\left(t_{4}, \tau\right)$ and $P_{a b}\left(t_{4}, \tau\right)$ of the transient decay following the last pump pulse depend on both quantities.

\section{Experiment}

The experiments were carried out with a double resonance MWFT-spectrometer which has already been described in [1] and [5]. The signal pulse was initiated by the experiment control unit. The pump pulse sequence was produced by a home-made TTL-double pulse generator which was triggered by the experiment control unit and initiated simultaneously with switch 17 of Fig. 1 in [5]. After each experiment consisting of a given number of averaging cycles the averaged decay was Fourier transformed and the intensity of the observed line was calculated. Then the time delay between both pump pulses was automatically incremented and the experiment was started again. When we plotted the measured intensities $I(\tau)$ as a function of the delay time $\tau$ between the pump pulses we obtained a sinusoidally modulated decay as it has been described theoretically. An example is given in Figure 2. The modulation frequency of the curve represents the off-resonance $\Delta \omega_{b c}$ of the pump. It is $2 \mathrm{MHz}$ in Figure 2. Because of a $10 \mathrm{MHz}$ clock-frequency of our experiment control unit we were up to now not able to use smaller increments than $100 \mathrm{~ns}$. As a

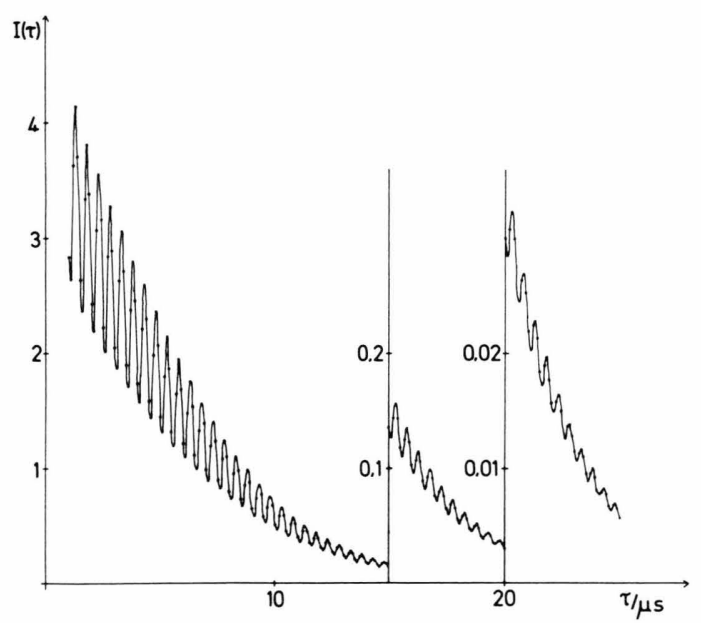

Fig. 2. Time-resolved response $I(\tau)$ of the $(2 \leftarrow 1)_{\mathrm{p}}-$ $(1 \leftarrow 0)_{\mathrm{s}}$ system of OCS as a function of the delay between the pump pulses. Measured points given by $\bullet$. Signal pulse frequency at $12162.975 \mathrm{MHz}$ (resonant), $3 \mathrm{~W}, 1.0 \mathrm{mTorr}$, $20{ }^{\circ} \mathrm{C}$, intensities in arbitrary units.

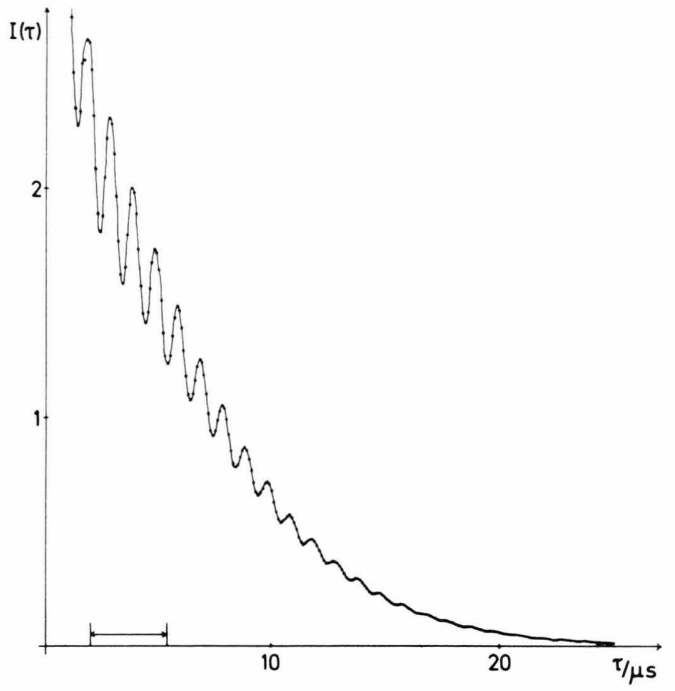

Fig. 3 a. Time resolved response of the $(2 \leftarrow 1)_{\mathrm{p}}-(1 \leftarrow 0)_{\mathrm{s}}$ system of OCS as a function of the delay $\tau$ between the pump pulses. The interpolated curve of the measured points - indicates a modulation frequency of $1 \mathrm{MHz}$. Signal pulse frequency at $12162.975 \mathrm{MHz}$ (resonant) pump pulses frequency at $24336.927 \mathrm{MHz}$ (11 MHz off-resonant), $3 \mathrm{~W}, 1.0 \mathrm{mTorr}, 20^{\circ} \mathrm{C}$, intensities in arbitrary units.

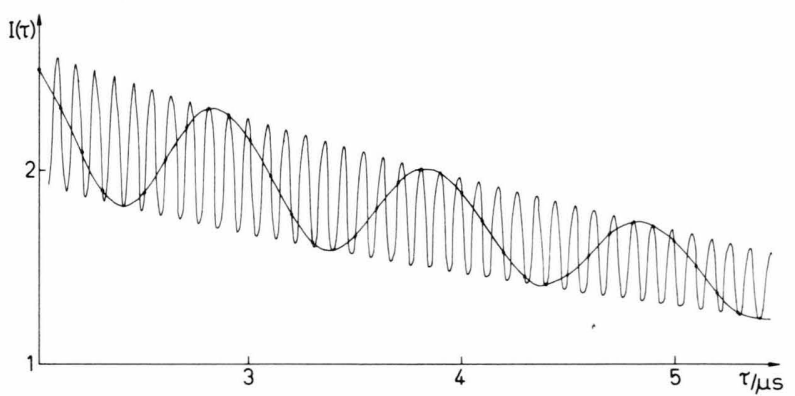

Fig. 3b. The interpolated curve with $1 \mathrm{MHz}$ modulation frequency $(-)$ is enlarged and supplemented by a curve with a modulation frequency of $11 \mathrm{MHz}$. In this case this is the response function $I(\tau)$ of the molecular system.

minimum of two data points is necessary for each period because of the sampling theorem, the maximum detectable off-resonance frequency is $5 \mathrm{MHz}$. To show that an off-resonance larger than $5 \mathrm{MHz}$ can be measured, we chose an off-resonance of $(10 n+1) \mathrm{MHz}, n=1,2,3$. At a sample rate of $100 \mathrm{~ns}$ in all these cases the actually present modulation frequency of $I(\tau)$ is folded to a frequency of $1 \mathrm{MHz}$ which can easily be measured. An example for a pump off-resonance of $11 \mathrm{MHz}$ is given in Figure 3. Figure 3 a shows a curve interpolating the 
measured values $I(\tau)$. This curve shows a modulation frequency of $1 \mathrm{MHz}$. In Fig. $3 \mathrm{~b}$ a section of Fig. 3a has been enlarged in order to show details of the sampling technique. The response $I(\tau)$ of the molecular ensemble corresponding to a curve with higher modulation frequency, which cannot be detected directly because of the limitations given by the sampling theorem, was drawn in subsequently.

\section{Conclusion}

We showed that a double resonance experiment with Fourier transform technique can be made in the form of a two-dimensional spectroscopy providing the frequency of a signal transition and the offresonance of a pump frequency. The two time dimensions are:

1. Time elapsing during the measured decay (phase 5),

2. Time interval $\tau$ between two pump pulses (phase 3).

[1] H. Dreizler, E. Fliege, H. Mäder, and W. Stahl, Z. Naturforsch. 37 a, 1266 (1982).

[2] D. Andrews, J. G. Baker, B. J. Blundell, and G. C. Petty, J. Mol. Struct. 97, 271 (1983).

[3] H. Bomsdorf and H. Dreizler, Z. Naturforsch. 36 a, 473 (1981).
So a successful search for double resonances to facilitate the assignment of rotational spectra should be possible.

Because of the sampling theorem the experiment should be modified to allow increments of the time interval between the two pump pulses of $10 \mathrm{~ns}$. Thus an off-resonance of $50 \mathrm{MHz}$ should be observable without folding.

To introduce the two-dimensional MWFT spectroscopy as a general tool more work is necessary.

\section{Acknowledgements}

We thank the members of our group for help and discussions. We are especially indebted to Dr. G. Bestmann for his assistance in digital electronics, to Dr. H. Mäder for critically reading the manuscript and to the workshop for manufacturing waveguide components. We further thank the Deutsche Forschungsgemeinschaft and Fonds der Chemie for funds.

[4] Doetsch: Anleitung zum praktischen Gebrauch der Laplace-Transformation, Verlag R. Oldenbourg, München.

[5] G. Bestmann and H. Dreizler, Z. Naturforsch. 38 a, 452 (1983). 